\title{
SCAMP5 Plays a Critical Role in Synaptic Vesicle Endocytosis during High Neuronal Activity
}

\author{
Haiyan Zhao, ${ }^{1,2}$ Yoonju Kim, ${ }^{1,2,3}$ Joohyun Park, ${ }^{1,2,3}$ Daehun Park, ${ }^{1,2}$ Sang-Eun Lee, ${ }^{1,2}$ Iree Chang, ${ }^{1}$ \\ and Sunghoe Chang ${ }^{1,2,3,4}$ \\ ${ }^{1}$ Department of Physiology and Biomedical Sciences, ${ }^{2}$ Biomembrane Plasticity Research Center, ${ }^{3}$ Neuroscience Research Institute, Medical Research \\ Center, and ${ }^{4}$ Bio-Max Institute, Seoul National University College of Medicine, Seoul, South Korea 110-799
}

Secretory carrier membrane protein 5 (SCAMP5), a recently identified candidate gene for autism, is brain specific and highly abundant in synaptic vesicles (SVs), but its function is currently unknown. Here, we found that knockdown (KD) of endogenous SCAMP5 by SCAMP5specific shRNAs in cultured rat hippocampal neurons resulted in a reduction in total vesicle pool size as well as in recycling pool size, but the recycling/resting pool ratio was significantly increased. SCAMP5 KD slowed endocytosis after stimulation, but impaired it severely during strong stimulation. We also found that KD dramatically lowered the threshold of activity at which SV endocytosis became unable to compensate for the ongoing exocytosis occurring during a stimulus. Reintroducing shRNA-resistant SCAMP5 reversed these endocytic defects. Therefore, our results suggest that SCAMP5 functions during high neuronal activity when a heavy load is imposed on endocytosis. Our data also raise the possibility that the reduction in expression of SCAMP5 in autistic patients may be related to the synaptic dysfunction observed in autism.

Key words: neuronal activity; recycling pool; resting pool; SCAMP5; synaptic vesicle endocytosis

\section{Introduction}

Secretory carrier membrane proteins (SCAMPs) are secretory vesicle components found in exocrine glands. Of the five currently known SCAMPs (SCAMPs 1-5), SCAMPs 1-3 share a common domain structure comprising a cytoplasmic $\mathrm{N}$-terminal domain with multiple endocytic NPF repeats, 4 highly conserved transmembrane regions, and a short cytoplasmic C-terminal tail. SCAMPs 4 and 5 lack the N-terminal NPF repeats and were thus thought not to function in endocytosis (Fernández-Chacón and Südhof, 2000).

SCAMPs 1-4 are expressed ubiquitously, whereas SCAMP 5 is known to be brain specific. SCAMPs 1 and 5 are highly abundant in synaptic vesicles (SVs; Fernández-Chacón and Südhof, 2000). SCAMPs 1-3 were shown to play a role in fusion pore formation at the plasma membrane during dense-core vesicle (DCV) secretion in PC12 cells and also in trafficking events in the trans-Golgi network (TGN) and endosomal recycling compartment, suggesting that their basic role is in vesicular trafficking ( $\mathrm{Wu}$ and Castle, 1998; Fernández-Chacón et al., 1999; Guo et al., 2002; Liu et al.,

Received May 28, 2014; revised June 16, 2014; accepted June 20, 2014.

Author contributions: H.Z., Y.K., and S.C. designed research; H.Z., Y.K., J.P., D.P., S.-E.L., and I.C. performed research; H.Z., Y.K., J.P., D.P., S.-E.L., I.C., and S.C. analyzed data; H.Z., Y.K., I.C., and S.C. wrote the paper.

This work was supported by the Biomembrane Plasticity Research Center funded by the National Research Foundation of Korea (Grant 20100029395 to S.C.).

The authors declare no competing financial interests.

Correspondence should be addressed to Sunghoe Chang, PhD, Department of Physiology, Seoul National Univer-

sity College of Medicine, \# 309 Biomedical Science Bldg., 28 Yeongeon-dong, Jongno-gu, Seoul 110-799, South Korea. E-mail: sunghoe@snu.ac.kr.

I. Chang's present address: Leibniz-Institut für Altersforschung, Fritz-Lipmann-Institut e.V. (FLI), Beutenbergstra $\beta$ e 11, 07745 Jena, Germany.

DOI:10.1523/JNEUROSCI.2156-14.2014

Copyright $\odot 2014$ the authors $\quad 0270-6474 / 14 / 3410085-11 \$ 15.00 / 0$
2002; Lin et al., 2005; Liu et al., 2005; Liao et al., 2007; Liao et al., 2008; Zhang and Castle, 2011).

Although the high levels of SCAMP1 expression in SVs suggested that it played a role in synaptic physiology, analysis of SCAMP1 knock-out mice showed that this protein was not essential for synaptic functions (Fernández-Chacón et al., 1999), raising the possibility that other, brain-specific SCAMPs such as SCAMP5 might be active. SCAMP5 is expressed only in the brain and is undetectable in neuroendocrine glands that express many other neuron-specific proteins such as synaptophysin and synaptotagmin (Fernández-Chacón and Südhof, 2000). This suggests a selective role for SCAMP5 in SV trafficking, but evidence for this is lacking. A recent study identified SCAMP5 as a candidate gene for autism and showed that it was silenced on a derivative chromosome and its expression was reduced to $<40 \%$ in a patient with idiopathic, sporadic autism (Castermans et al., 2010). Therefore, the reduction in the expression of SCAMP5 may be related to the synaptic dysfunction observed in autistic patients.

In the present study, we found that knockdown (KD) of endogenous SCAMP5 by SCAMP5-specific shRNAs led to a reduction in both total vesicle pool size and recycling pool size and the recycling/resting pool ratio was increased significantly. The defect in SV endocytosis was mainly apparent during strong stimulation. Therefore, our results suggest that SCAMP5 functions in controlling the SV recycling machinery during high levels of neuronal activity.

\section{Materials and Methods}

DNA constructs. SCAMP5 (GeneID: 65171) was purchased from SuperScript rat brain cDNA library (Invitrogen), amplified by PCR, and subcloned into pEGFP (Clontech) or mCherry (generously provided by Dr. 
A

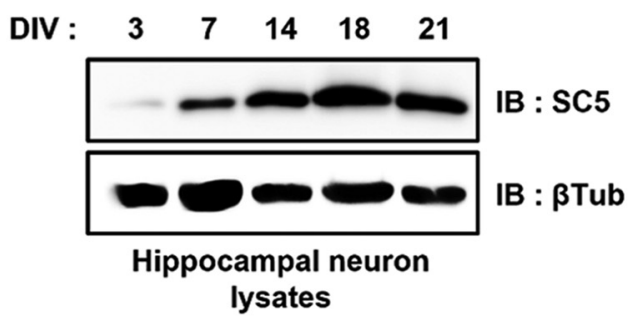

B1

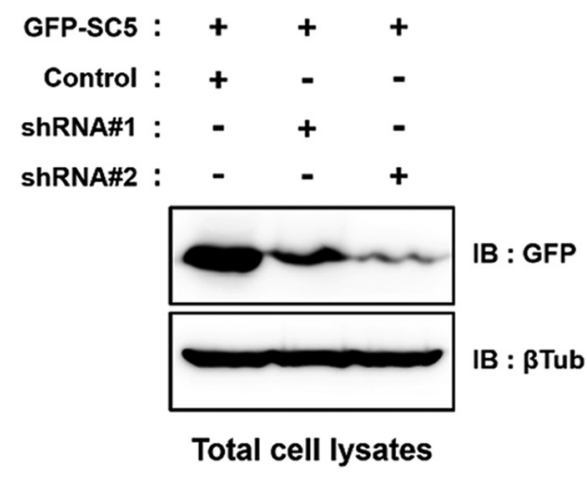

B2

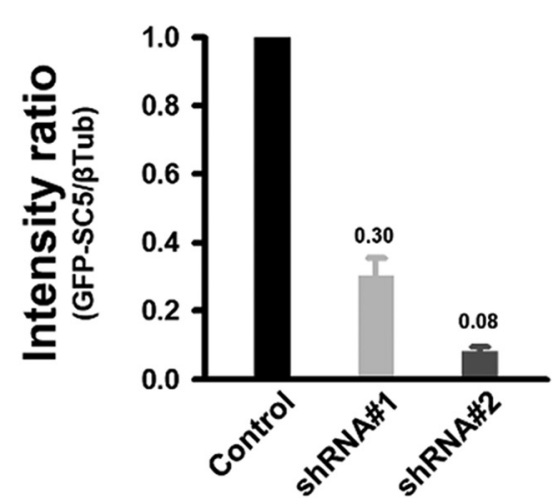

C

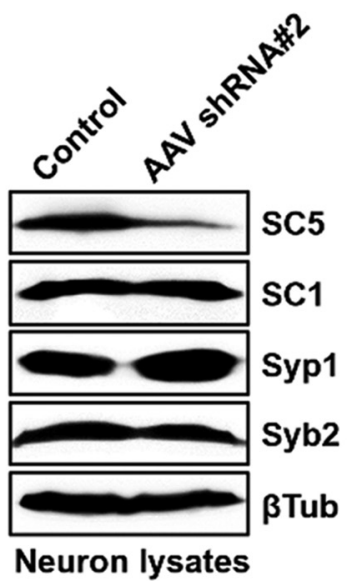

Figure 1. Expression pattern of SCAMP5 protein during development of neuron in culture and suppression of SCAMP5 expression by shRNAs. A, Developmental changes of SCAMP5 protein expression in cultured rat hippocampal neurons. Cultured primary hippocampal neurons were prepared at the indicated days in vitro (DIV), lysed, and evenly loaded (75 $\mu$ g) in SDS-PAGE gels. Western blot analysis was performed using a specific anti-SCAMP5 antibody. SC5, SCAMP5; $\beta$ Tub, $\beta$-tubulin. SCAMP5 expression rises during DIV 3 - 14 and then the levels persist. B1-B2, HEK293T cells were cotransfected with EGFP-SCAMP5 and pU6mRFP-vector (control) or pU6mRFP-SCAMP5-specific shRNAs, respectively. Then, $72 \mathrm{~h}$ after transfection, the cells were lysed and evenly loaded $(25 \mu \mathrm{g})$ in SDS-PAGE. Western blot analysis was performed using anti-GFP antibody and anti- $\beta$ Tub antibody. Both SCAMP5-targeted shRNAs efficiently knocked down the expression of GFPSCAMP5 (expression levels over control: $0.3 \pm 0.04$ for shRNA\#1, $n=4,0.08 \pm 0.01$ for shRNA\#2, $n=4$ ). C, Immunoblot analysis of primary cultured neurons infected with control or SCAMP5 shRNA\#2-based AAV (SC5 shRNA\#2) for endogenous SCAMP5 KD. The cells were infected at DIV 7 and were lysed at DIV 21. Western blot analysis was performed using the indicated specific antibodies. SC1, SCAMP1; Syp1, synaptophysin 1; Syb2, synaptobrevin 2; $\beta$ Tub, $\beta$-tubulin.

Roger Y. Tsien at University of California-San Diego) vector. The fidelity of all constructs was verified by sequencing. vGlut1-pHluorin (vGpH), synaptophysin-pHluorin (SypHy), and synaptopHluorin were kindly provided by Dr. John Rubenstein at University of California-San Francisco, Dr. Leon Lagnado at the Medical Research Council, and Dr. James Rothman at Sloan Kettering Cancer Center, respectively.

Antibodies and reagents. The following antibodies were used: antiSCAMP5 antibody that does not recognize SCAMP1 was from Sigma (catalog \#S0943); anti-SCAMP1 antibody (catalog \#121002), antisynaptophysin antibody (catalog \#101011), and anti-synaptobrevin 2 antibody (catalog \#104211) were from SYSY; anti-tubulin antibody, antiGFP antibody, and anti- $\beta$ tubulin antibody were from Abcam. Secondary antibodies were obtained from Jackson ImmunoResearch. Bafilomycin A1 was from Calbiochem and all other reagents were from Sigma.

Neuron culture and transfection. Hippocampal neurons derived from embryonic day 18 Sprague Dawley fetal rats of either sex were prepared as described previously (Chang and De Camilli, 2001). Briefly, hippocampi were dissected, dissociated with papain, and triturated with a polished half-bore Pasteur pipette. The cells $\left(2.5 \times 10^{5}\right)$ in minimum Eagle's medium (Invitrogen), supplemented with $0.6 \%$ glucose, $1 \mathrm{~mm}$ pyruvate, $2 \mathrm{~mm}$ L-glutamine, $10 \%$ fetal bovine serum (Hyclone), and antibiotics, were plated on poly-D-lysine-coated glass coverslips in a $60 \mathrm{~mm}$ Petri dish. Four hours after plating, the medium was replaced with neurobasal medium (Invitrogen) supplemented with 2\% B-27, 0.5 mM L-glutamine; $4 \mu \mathrm{M} 1-\beta$-D-cytosine-arabinofuranoside (Ara-C; Sigma) was added as needed. Neurons were transfected using a modified calcium-phosphate method (Lee et al., 2006). Briefly, $6 \mu \mathrm{g}$ of cDNA and $9.3 \mu \mathrm{l}$ of $2 \mathrm{M} \mathrm{CaCl}_{2}$ were mixed in distilled water to a total volume of $75 \mu \mathrm{l}$ and the same volume of $2 \times$ BBS was added. The cell culture medium was completely replaced by transfection medium (MEM; $1 \mathrm{~mm}$ pyruvate, $0.6 \%$ glucose, $10 \mathrm{~mm}$ glutamine, and $10 \mathrm{~mm}$ HEPES, $\mathrm{pH} 7.65$ ), and the cDNA mixture was added to the cells and incubated in a $5 \% \mathrm{CO}_{2}$ incubator for $90 \mathrm{~min}$. Cells were washed twice with washing medium, $\mathrm{pH} 7.35$, and then returned to the original culture medium. vGpH or SypHy and pU6mRFP constructs were cotransfected in a ratio of 5:1.

SCAMP5 KD. SCAMP5-specific small hairpin RNA (shRNA) was designed from the rat SCAMP5 cDNA sequence (NM_031726) targeting the region of nucleotides 5'-GCCATGTTTCTACCAAGACTT-3' ( shRNA\#1, nucleotides 54-74) and 5'-GCATGGTTCATAAGTT CTA-3' (shRNA\#2, nucleotides 509-527). A pair of complementary oligonucleotides was synthesized separately with the addition of an ApaI enzyme site at the $5^{\prime}$ end and an EcoRI site at the $3^{\prime}$ end. The annealed cDNA fragment was cloned into the ApaI-EcoRI sites of pSilencer 1.0-U6 vector (Ambion) modified by inserting an $\mathrm{mRFP}$ at the $\mathrm{C}$ terminus. For evading RNA interference, silent mutations within shRNA\#2 targeting sequence (T516C, T519C, and C525T) in HA-SCAMP5 were generated using the QuikChange Site-Directed Mutagenesis Kit (Stratagene). The fidelity of all constructs was verified by sequencing. shRNA\#2 sequences was cloned into the pAAV-U6 shRNA vector using BamHI/SalI sites and adeno-associated virus (AAV) vectors were produced by the KIST virus facility (Seoul, Korea) by cotransfecting each pAAV vector with the pAAV-RC1 and pHelper vector (Cell Biolabs) in the 293FT packaging 
A1

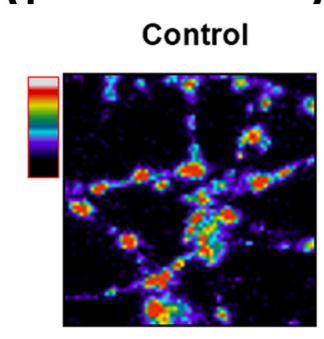

A2

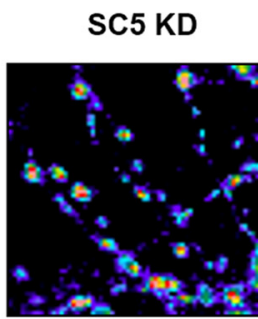

A3

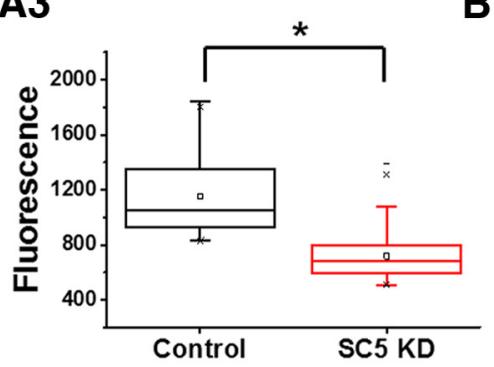

B

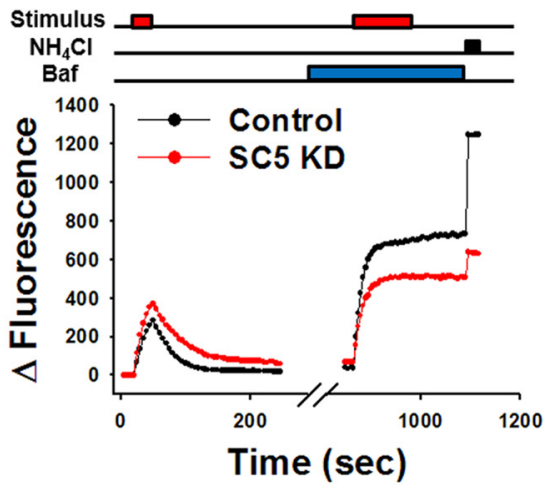

C3

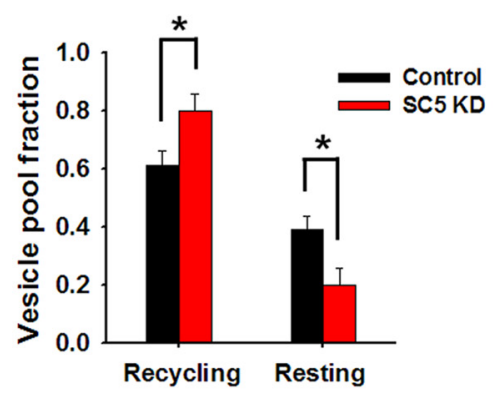

D3

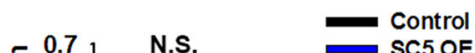

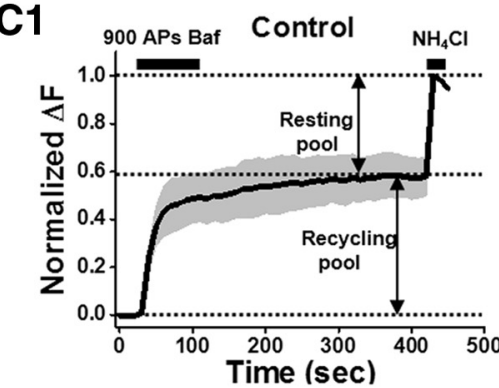

D1

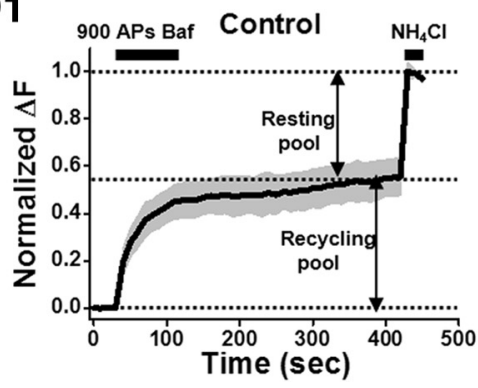

C2

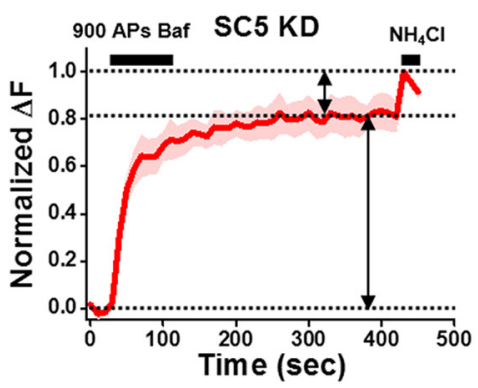

D2

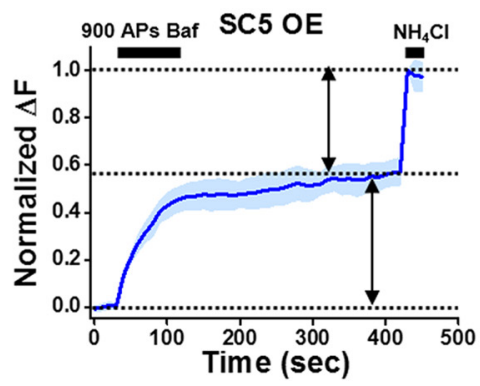

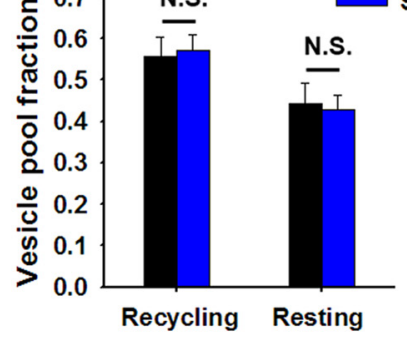

Figure 2. Suppression of SCAMP5 expression resulted in a reduction of total and recycling SV pool size, but the ratio of recycling/resting pool size was significantly increased with SCAMP5 KD. A1-A2, Representative fluorescence images of control and $\mathrm{KD}$ neurons transfected with $\mathrm{vGpH}$ and treated with $\mathrm{NH}_{4} \mathrm{Cl}$ to reveal total vesicle pool size of given boutons. Pseudocolor indicates relative fluorescence intensities. A3, Box-and-whiskers graph of total vesicle pool size (median: 1054.098 and 682.936; upper and lower quartiles: upper 1347.681 and 792.491, lower 925.049 and 596.255; maximum values: 1843.495 and 1385.644; minimum values: 828.552 and 502.826 for control and SCAMP5 KD, respectively. Open circle indicates mean: $1152.09 \pm 17.33, n=5$ for control, $717.64 \pm 10.06, n=6$ for KD. $\boldsymbol{B}$, Representative pooled average VGpH profiles for comparison of recycling pool size and total vesicle pool size between control and SCAMP5 KD synapses. Neurons were stimulated with $300 \mathrm{APs}$ at $10 \mathrm{~Hz}$ without Baf. After 10 min resting period, neurons were stimulated with $900 \mathrm{APs}$ at $10 \mathrm{~Hz}$ in the presence of $B$ af. The change in fluorescence intensity to the plateau reflects the entire recycling pool. All remaining acidic vesicles were alkalized by $\mathrm{NH}_{4} \mathrm{Cl}$ treatment, revealing the size of the resting pool. Due to variation in bouton size, even in an individual neuron, we chose to compare the similar size of boutons from control and KD neurons ( $n=25$ for control, 25 for KD). C1-C2, Averaged time course of $v G$ pH fluorescence traces in the presence of Baffollowed by $\mathrm{NH}_{4} \mathrm{Cl}$ treatment. The absolute amplitude of the signal differs from bouton to bouton due to variation in vesicle pool size, even in an individual neuron, so we normalized the each pool size to the total vesicle pool size (i.e., to the maximum fluorescence change after $\mathrm{NH}_{4} \mathrm{Cl}$ treatment), providing a signal that is independent of vesicle pool size.( $n=12 \mathrm{for}$ control, 14 for $\left.\mathrm{KD}\right) \mathrm{C}$, Average fraction values of recycling: resting pool for control, and SCAMP5 KD synapses ( $0.61 \pm 0.04: 0.39 \pm 0.04$ for control vs $0.80 \pm 0.05: 0.19 \pm 0.05$ for KD). Data are presented as means \pm SE. ${ }^{*} p<0.01$ (Student's t test). D1-D2, Averaged time course of vGpH fluorescence traces in control and SCAMP5-overexpressed (0E) synapses $(n=11$ for control, 13 for 0E). D3, Average fraction values of recycling: resting pool for control, and SCAMP5-overexpressed synapses ( $0.56 \pm 0.04: 0.44 \pm 0.04$ for control vs $0.58 \pm 0.03: 0.42 \pm 0.03$ for $0 \mathrm{E})$. Data are presented as means \pm SE. ${ }^{*} p<$ 0.01 (Student's $t$ test).

cell line. The supernatant was collected and concentrated by ultracentrifugation.

KD efficiency was examined in GFP-SCAMP5-expressed HEK293T cells or AAV-shRNA\#2-infected cultured hippocampal neurons by Western blotting. HEK293T cells were cultured at $37^{\circ} \mathrm{C}$ and $5 \% \mathrm{CO}_{2}$ in DMEM (Invitrogen) supplemented with 10\% fetal bovine serum and transfected with GFP-SCAMP5 and shRNAs using Lipofectamine 2000 (Invitrogen). Cells were examined for transfection efficiency after 16-24 h under a fluorescence microscope. For Western blotting, HEK293T cells or AAV-infected hippocampal neurons were lysed in a lysis buffer containing the following (in $\mathrm{mm}$ ): 1 sodium orthovanadate, $10 \mathrm{NaF}, 10$ Tris$\mathrm{HCl}, \mathrm{pH}$ 7.4, 1 PMSF, 10 leupeptin, 1.5 pepstatin, and 1 aprotinin, along with $1 \%$ SDS, clarified by centrifugation $15,000 \times g$ for $10 \mathrm{~min}$, and incubated for $15 \mathrm{~min}$ in $37^{\circ} \mathrm{C}$ water bath. Protein concentrations were measured with a bicinchoninic acid protein assay reagent kit (Thermo
Fisher Scientific). Samples containing $100 \mu \mathrm{g}$ of total protein were separated by SDS-PAGE and transferred to PVDF membranes (Bio-Rad). The membranes were blocked for $1 \mathrm{~h}$ with 5\% nonfat dry milk in TBST (10 mm Tris-HCl, pH 7.5,100 mm NaCl, and 0.1\% Tween 20) incubated with the respective primary antibodies for $2 \mathrm{~h}$ at room temperature. After extensive washing in TBST, the membrane was incubated with horseradish peroxidase-conjugated secondary antibody (Jackson ImmunoResearch). The antigen-antibody complexes were detected with enhanced chemiluminescence reagents (Abclon). shRNA\#2 was used to knock down the expression of SCAMP5 in all of the experiments except for those shown in Figure $4 C$, in which shRNA\#1 was used.

Synaptic vesicle pool size measurement. To estimate the size of each fraction of the SV pool, vGpH-transfected neurons at $16 \mathrm{~d}$ in vitro were stimulated with 900 action potentials (APs) at $10 \mathrm{~Hz}$ in the presence of $0.5 \mu \mathrm{m}$ bafilomycin A1 (Baf) to release the entire recycling pool of SVs 
A

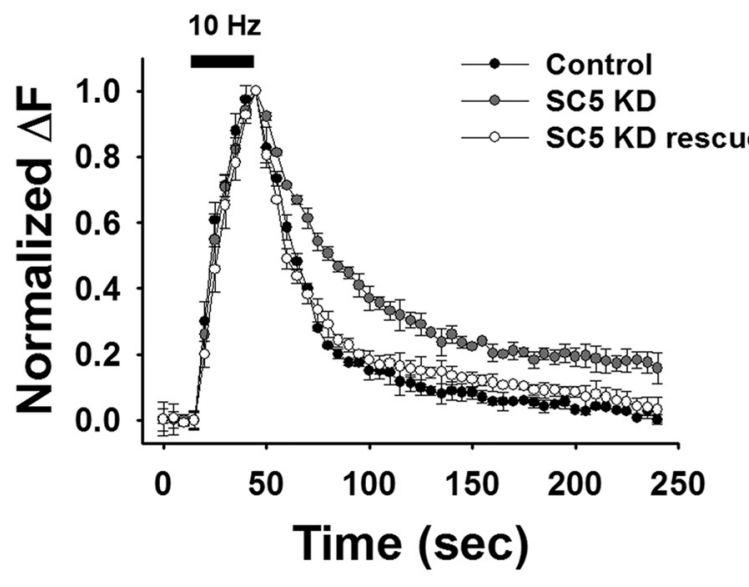

B

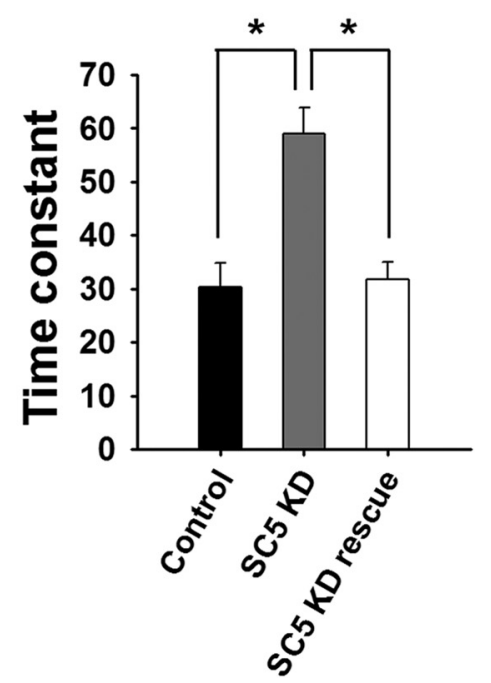

Figure 3. KD of endogenous SCAMP 5 slowed endocytosis after stimulation. $A$, Average $\mathrm{VGpH}$ fluorescence intensity profiles, plotted as $\Delta F / F_{0}$ against time, after stimulation with $300 \mathrm{APs}$ at 10 $\mathrm{Hz}$ (dark bar). Fluorescence values were normalized to the maximal fluorescence signal in each experimental condition. $B$, Decay of $\mathrm{vGpH}$ after stimulation fitted by a single exponential with time constant, $\tau=30.28 \pm 4.51 \mathrm{~s}$ for control $(n=8), 58.96 \pm 4.82 \mathrm{~s}$ for SCAMP5 KD $(n=9), 31.75 \pm 3.19$ s for SCAMP5 KD rescue $(n=9)$. Data are presented as means \pm SE. ${ }^{*} p<0.01$ (ANOVA and Tukey's HSD post hoc test).

(Burrone et al., 2006). Baf was dissolved in $\mathrm{Me}_{2} \mathrm{SO}$ to $0.2 \mathrm{~mm}$ and diluted to a final concentration of $0.5 \mu \mathrm{m}$ before the experiments. Baf was applied throughout the experiments. The change in fluorescence intensity to the plateau reflects the entire recycling pool. The resting pool that cannot be mobilized by neuronal activity can be uncovered by applying $\mathrm{NH}_{4} \mathrm{Cl}$ solution to unquench all acidic vesicles that have not been released. Fluorescence intensity was normalized to the maximum fluorescence change after $\mathrm{NH}_{4} \mathrm{Cl}$ treatment. Data were collected from $30-40$ boutons of 12-24 neurons in each coverslip and " $n$ " stands for the number of coverslip. Data are presented as means \pm SE. Statistical analysis was performed with SPSS Version 19 software. For multiple conditions, means were compared by ANOVA followed by Tukey's HSD post hoc test.

$v \mathrm{GpH}$ (or SypHy) exo/endocytosis assay and image analysis. Coverslips were mounted in a perfusion/stimulation chamber equipped with platinum-iridium field stimulus electrodes (Chamlide; LCI) on the stage of an Olympus IX-71 inverted microscope with $40 \times, 1.0$ numerical aperture oil lens. The cells were continuously perfused at room temperature with Tyrode solution containing the following (in $\mathrm{mm}$ ): $136 \mathrm{NaCl}, 2.5$ $\mathrm{KCl}, 2 \mathrm{CaCl}_{2}, 1.3 \mathrm{MgCl}_{2}, 10$ HEPES, and 10 glucose, $\mathrm{pH} 7.3 ; 10 \mu \mathrm{M}$ 6-cyano-7-nitroquinoxaline-2,3-dione was added to the imaging buffer to reduce spontaneous activity and to prevent recurrent excitation during stimulation. Time-lapse images were acquired every $5 \mathrm{~s}$ for $4 \mathrm{~min}$ using a back-illuminated Andor iXon 897 EMCCD camera driven by MetaMorph Imaging software (Molecular Devices). From the fourth frame, the cells were stimulated ( $1 \mathrm{~ms}, 20-50 \mathrm{~V}$, bipolar) using an A310 Accupulser current stimulator (World Precision Instruments). Quantitative measurements of the fluorescence intensity at individual boutons were obtained by averaging a selected area of pixel intensities using ImageJ. Individual regions were selected by hand, rectangular regions of interest were drawn around the synaptic boutons, and average intensities were calculated. Large puncta, typically representative of clusters of smaller synapses, were rejected during the selection procedure. The center of intensity of each synapse was calculated to correct for any image shift over the course of the experiment. Fluorescence was expressed in intensity units that correspond to fluorescence values averaged over all pixels within the region of interest. All fitting was done using individual error bars to weight the fit using Origin 8 (OriginLab). To obtain the endocytic time constant after stimulation, the decay of vGpH after stimulation was fitted with a single exponential function. In some experiments in which fluorescence decay does not decay to zero (as in the case of SCAMP5 KD), the time constant was obtained using the initial slope method. In this method, a line is drawn from the initial point at the initial slope and where that line intersects the final value is the time constant.

For exocytosis assays, neurons were preincubated with Baf for $60 \mathrm{~s}$ to block the reacidification and stimulated for $120 \mathrm{~s}$ at $10 \mathrm{~Hz}$, which is known to deplete total recycling pool of vesicles (Sankaranarayanan et al., 2000; Burrone et al., 2006). Net fluorescence changes were obtained by subtracting the average intensity of the first four frames $\left(F_{0}\right)$ from the intensity of each frame $\left(F_{\mathrm{t}}\right)$ for individual boutons, normalizing to the maximum fluorescence intensity $\left(F_{\max }-F_{0}\right)$, and then averaging. To get endocytic rate during stimulation, neurons were stimulated in the presence or absence of Baf. In the absence of Baf, the fluorescence signal reflects the net balance of exocytosis and endocytosis $\left(\Delta F_{\text {exo }}-\right.$ endo $)$. In the presence of $B a f$, exocytosis events are trapped in an alkaline state and the fluorescence signal reflects exocytosis $\left(\Delta F_{\text {exo }}\right)$. Fluorescence values were normalized to the peak fluorescence in each experimental condition. Endocytosis during stimulation was derived by subtracting the $\mathrm{vGpH}$ or SypHy fluorescence in the absence of Baf from that in the presence of $\operatorname{Baf}\left(\Delta F_{\text {endo }}=\Delta F_{\text {exo }}-\Delta F_{\text {exo }}\right.$ - endo $)$. The traces were normalized to the maximum stable fluorescence signal after $B a f$ treatment. Rate of exocytosis and rate of endocytosis during stimulation were obtained from the linear fits to the data during a 300 AP stimulus. Photobleaching drift was corrected empirically using either local background or time-lapse imaging without stimulation before the experiments. Because both yielded similar results (and actually prebleaching sometimes damaged the cells), we only used the local background method throughout the study. We selected the regions where blurred fluorescence signals are observed and those where no active changes in fluorescence intensity were observed during experiments. We took decay kinetics of these local backgrounds by fitting a double exponential function to local background decay signal and then subtracting this function from the original trace. The result was a trace with a relatively flat baseline. Data were collected from 30-40 boutons of 12-24 neurons in each coverslip and " $n$ " stands for the number of coverslips. Statistical analysis was performed with SPSS Version 19. For multiple conditions, we compared means by ANOVA followed by Tukey's HSD post hoc test or Fisher's LSD test (depending on the number of groups). For acidification assay, neurons were mounted in a rapid perfusion chamber (Chamlide; LCI) equipped with platinum-iridium electrodes. The extracellular buffer was changed twice from $\mathrm{pH} 7.4$ to 5.3 and back before and after $300 \mathrm{APs}$ at $10 \mathrm{~Hz}$.

FM1-43 uptake assay. Pools of synaptic vesicles were labeled during electrical stimulation for $30 \mathrm{~s}$ at $10 \mathrm{~Hz}$ in the presence of $10 \mu \mathrm{M}$ 
A1

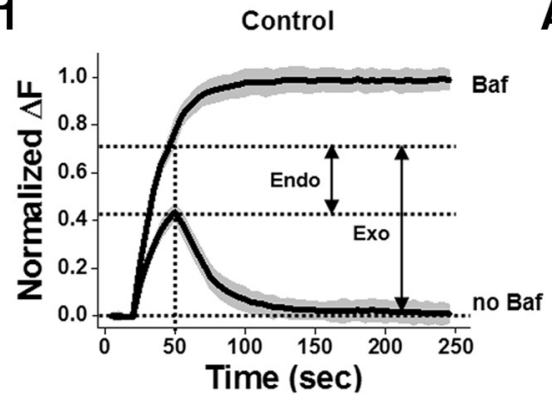

B1

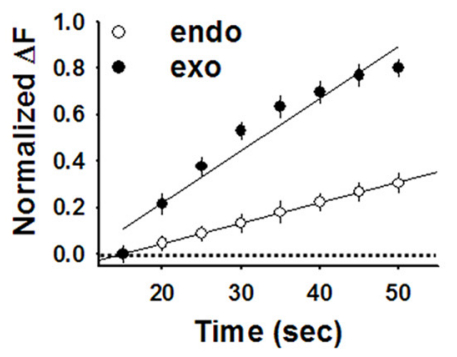

A2

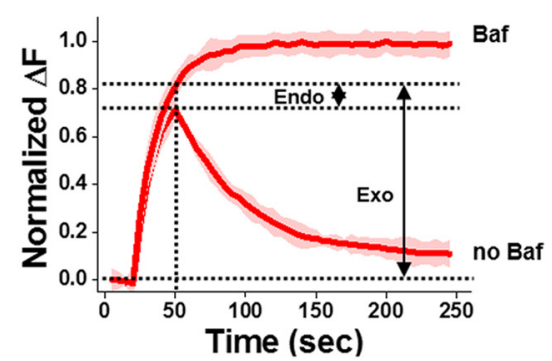

B2

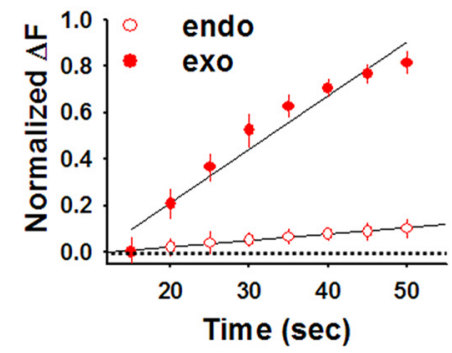

A3

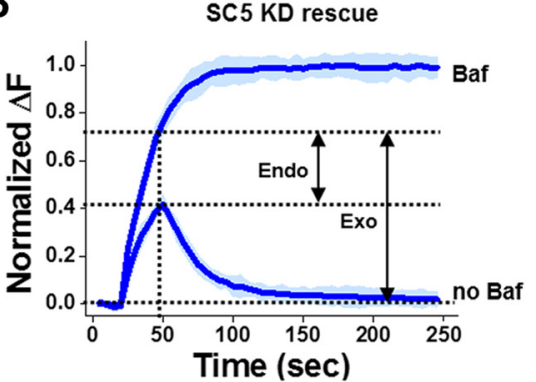

B3

SC5 KD rescue

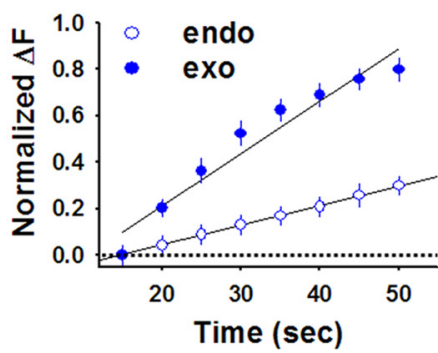

B4

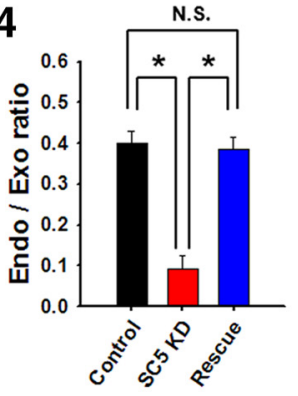

C1

C2
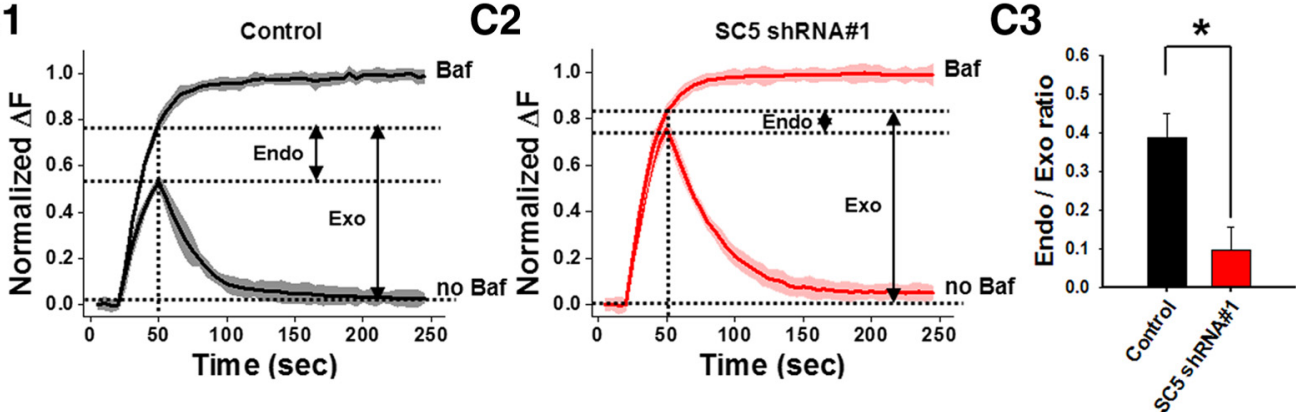

Figure 4. The KD ofSCAMP5 severelyimpaired SV endocytosis during stimulation. $\boldsymbol{A} 1-\mathbf{A 3}$, Average vGpH fluorescenceintensity profilesfrom control (A1), KD (A2), and rescued (A3) synapses stimulated with or without Baf. Neurons were stimulated with $300 \mathrm{APs}$ at $10 \mathrm{~Hz}$ without Baf. After a $10 \mathrm{~min}$ resting period, the same neurons were stimulated again with $1200 \mathrm{APs}$ at $10 \mathrm{~Hz}$ in the presence of Baf. Fluorescence values were normalized to the maximal fluorescence signal in the Baftrace ( $n=15$ for control, 15 for KD, 15 for rescue). $\boldsymbol{B}$ 1-B3, Graphs showing the time course of endocytosis (Endo) and exocytosis (Exo) during stimulation with $300 \mathrm{APs}$ at $10 \mathrm{~Hz}$. Time course of endocytosis during stimulation was derived by subtracting the fluorescence trace in the absence of $B a f\left(\Delta F_{\text {exo }}-\right.$ endo $)$ from the trace in its presence $\left(\Delta F_{\text {endo }}=\right.$ $\Delta F_{\text {exo }}-\Delta F_{\text {exo }- \text { endo }}$ ). Rate of exocytosis and rate of endocytosis during stimulation were obtained from the linear fits to the initial 30 s of traces (exocytic rate: $0.113 \pm 0.03$ for the control, $0.114 \pm 0.04$ for SCAMP5 KD, and $0.113 \pm 0.05$ for SCAMP5 rescue; endocytic rate: $0.046 \pm 0.02$ for the control, $0.010 \pm 0.04$ for SCAMP5 KD, and $0.043 \pm 0.03$ for SCAMP5 rescue). B4, Ratios of endocytosis/exocytosis rate: $0.402 \pm 0.03$ for the control, $0.092 \pm 0.04$ for SCAMP5 KD, and $0.386 \pm 0.03$ for SCAMP5 rescue. Data are presented as means \pm SE. ${ }^{*} p<0.01$ (ANOVA and Tukey's HSD post hoctest). C1-C3, SCAMP5 KD by another independent shRNA (shRNA\#1 in Fig. 1) also resulted in a severe defect in the endocytosis during stimulation. The average endo/exo ratio $(0.386 \pm 0.06, n=8$ for the control, $0.097 \pm 0.05, n=10$ for SCAMP5 KD) during 300 APs at $10 \mathrm{~Hz}$. Data are presented as means \pm SE. ${ }^{*} p<0.01$ (ANOVA and Tukey's HSD post hoc test).

FM1-43 (Invitrogen). FM1-43 was loaded with the onset of stimulation (300 APs) and immediately washed out with the cessation of stimulation. After $10 \mathrm{~min}$ of resting period, $1200 \mathrm{APs}$ at $10 \mathrm{~Hz}$ were given to unload and measure the amount of loaded FM1-43. The same neurons were stimulated again in the presence of FM1-43 and kept in the presence of dye for an additional $30 \mathrm{~s}$ after stimulation to label poststimulus endocytosed vesicles. After $10 \mathrm{~min}$ of resting period, $1200 \mathrm{APs}$ at $10 \mathrm{~Hz}$ were given to unload and measure the amount of loaded FM1-43. Fully unloaded images were taken after each unloading. Net fluorescence changes were obtained by subtracting the intensity of the unloaded image from the intensity of the loaded image.

\section{Results}

We used a specific SCAMP5 antibody to examine the expression of SCAMP5 by Western blot analysis in cultured hippocampal neurons. SCAMP5 was expressed in hippocampal neurons and its expression levels increased as the neurons matured (Fig. 1A).

To gain quantitative insight into the effect of reduced SCAMP5 expression on SV trafficking, we used two independent
shRNA constructs. Suppression of SCAMP5 expression was confirmed in HEK293T cells cotransfected with EGFP-SCAMP5 and SCAMP5-shRNA. The two shRNAs reduced SCAMP5 expression to $<35 \%$ ( shRNA\#1) and $<10 \%$ (shRNA\#2) of the original levels, respectively (Fig. 1B). The efficiency of shRNA\#2 construct was confirmed by AAV-mediated KD of endogenous SCAMP5 in neurons. Western blot results showed substantial reduction of SCAMP5 expression, whereas the expressions of SCAMP1 and other synaptic vesicle proteins, such as synaptophysin and synaptobrevin-2, were not affected (Fig. 1C).

To gain an insight into the effect of SCAMP5 KD on presynaptic function, neurons were cotransfected with $\mathrm{vGpH}$ and shRNA. vGpH is a vesicular glutamate transporter-1 fused with pHluorin, a modified GFP with high $\mathrm{pH}$ sensitivity (Sankaranarayanan et al., 2000; Voglmaier et al., 2006; Balaji and Ryan, 2007) the fluorescence of which is quenched in acidic conditions and increased in basic conditions within the lumen of SVs and upon exocytosis to the extracellular space. 
A

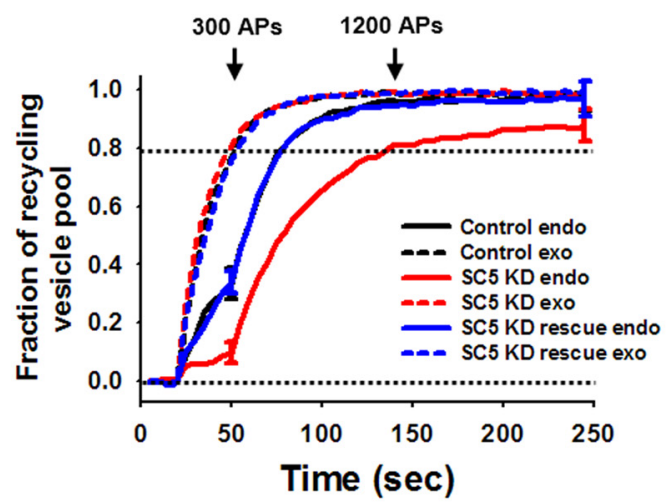

C1
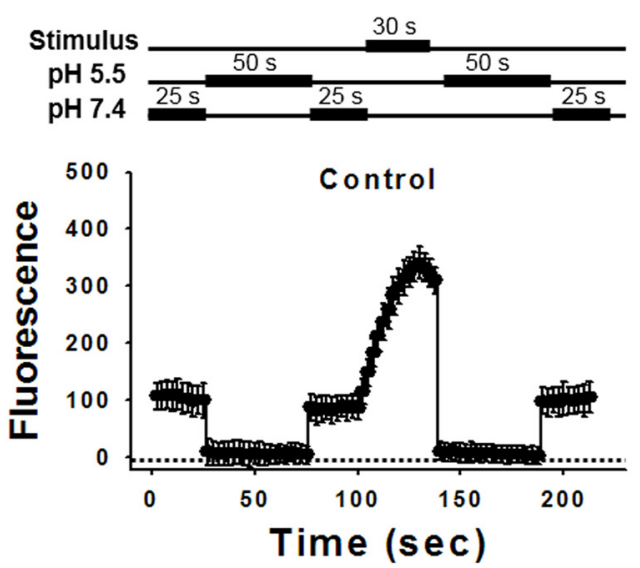

C2

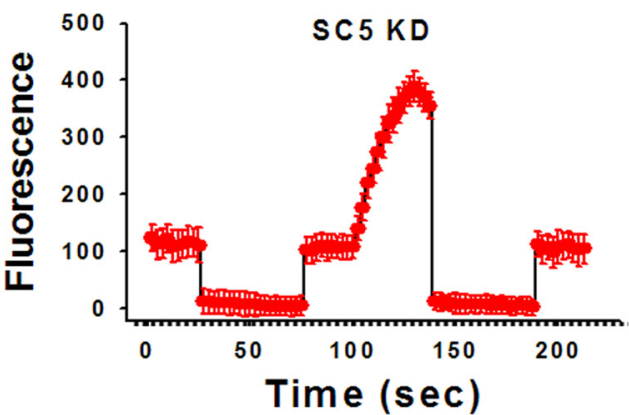

B

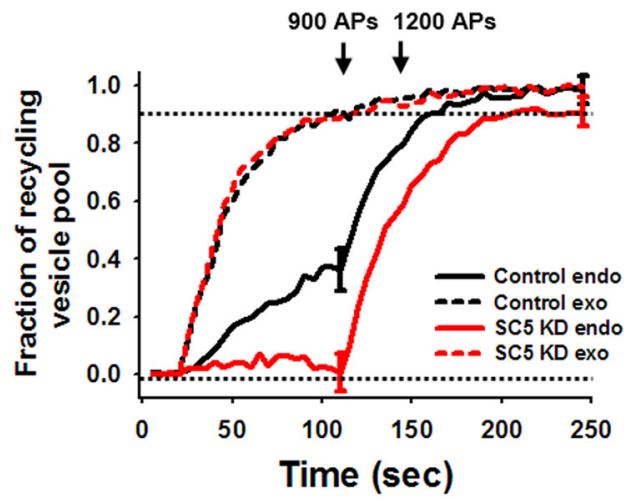

C3

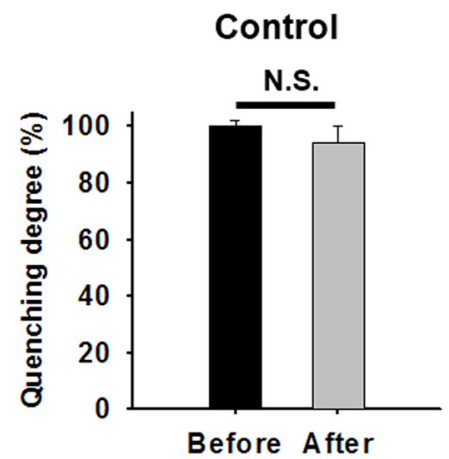

C4

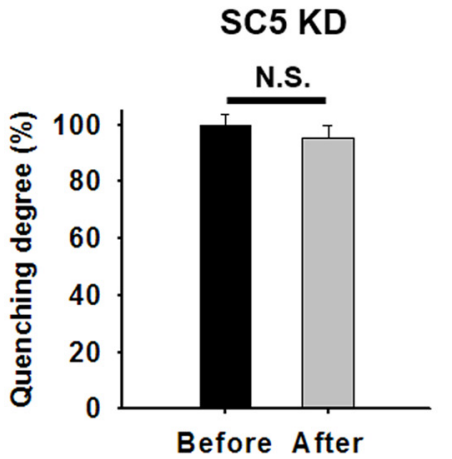

Figure 5. Average time courses of endocytosis clearly revealed severe defects in endocytosis during stimulation by SCAMP5 KD. A, Average time course of endocytosis from control (black), KD (red), and rescue (blue) neurons during and after $300 \mathrm{APs}$ at $10 \mathrm{~Hz}$ without Baf and the average time course of exocytosis from control (black dotted), KD (red dotted), and rescue (blue dotted) neurons during $1200 \mathrm{APs}$ at $10 \mathrm{~Hz}$ with Baf. A composite graph of the average time course of total endocytosis was obtained by combining the time course of endocytosis during stimulation [i.e., ( + )Baf $(-) B a f$ ) with the inverse image of the time course of endocytosis after stimulation in Figure $4, A 1-A 3$. The dashed line indicates the extent of exocytosis at the 30 s time point where the endocytosis to exocytosis ratios is calculated. Error bars are shown at two time points on the endocytosis curves. $B$, Average time course of endocytosis from control (black) and $K D$ (red) neurons during and after $900 \mathrm{APs}$ at $10 \mathrm{~Hz}$ without Baf and the average time course of exocytosis from control (black dotted) and KD (red dotted) neurons during $1200 \mathrm{APs}$ at $10 \mathrm{~Hz}$ with Baf. Error bars are shown at two time points on the endocytosis curves. C1-C2, Average traces of synaptopHluorin signal in control and SCAMP5 KD during pH exchange experiments. The extracellular solution is changed twice from pH 7.4 to 5.5 and back. Extracellular application of pH 5.5 Tyrode solution rapidly quenches all surface sPH in the prestimulus period. After $50 \mathrm{~s}$, the extracellular solution is changed back to $\mathrm{pH}$ 7.4. After a $30 \mathrm{~s} \mathrm{stimulus} \mathrm{at} 10 \mathrm{~Hz}$, the extracellular solution is changed to $\mathrm{pH} 5.5$ for $50 \mathrm{~s}$ then back to $\mathrm{pH}$ 7.4. Net $\mathrm{sPH}$ fluorescence changes were obtained by subtracting the

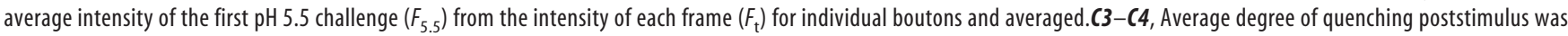
$94.11 \pm 5.88 \%$ in control $(n=5)$ and $95.11 \pm 4.88 \%$ in $\mathrm{KD}(n=6)$.

An SV pool is made up of a recycling pool consisting of a readily releasable pool and a reserve pool and a resting pool that does not normally recycle (Murthy and De Camilli, 2003; Rizzoli and Betz, 2004, 2005; Denker and Rizzoli, 2010). The total recycling pool is defined as the amplitude of the response to 900 APs at $10 \mathrm{~Hz}$ in the presence of Baf, a V-type ATPase inhibitor that blocks the acidification of endocytosed SVs (Sankaranarayanan and Ryan, 2000; Burrone et al., 2006). The resting pool of vesicles refractory to stimulation is uncovered by adding $\mathrm{NH}_{4} \mathrm{Cl}$, which traps all of the vesicles in an alkaline state (Sankaranarayanan and Ryan, 2000; Burrone et al., 2006).
We found that SCAMP5 KD resulted in a reduction of the total vesicle pool size (mean arbitrary fluorescence intensity: $1152.09 \pm 17.33$ for control, $717.64 \pm 10.06$ for KD; Fig. $2 A 1-$ A3). The absolute amplitude of the $\mathrm{vGpH}$ signal differs from bouton to bouton due to variations in bouton size and release probability, even in individual neurons (Murthy et al., 1997; Trommershäuser et al., 2003). However, when we compared the pooled average amplitude of the signal following 900 APs in the presence of $B a f$ with that from similar size of control boutons, it was evident that the recycling pool size was also reduced in SCAMP5 KD cells (Fig. 2B). To provide a signal that is indepen- 
A1

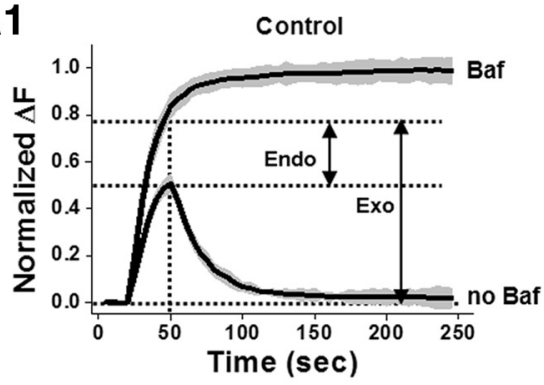

B

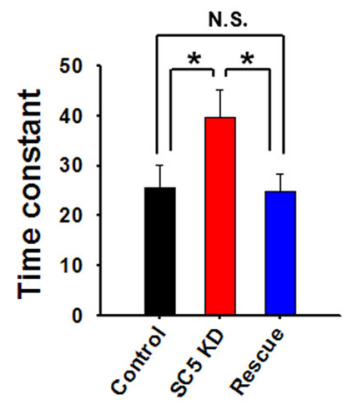

A2

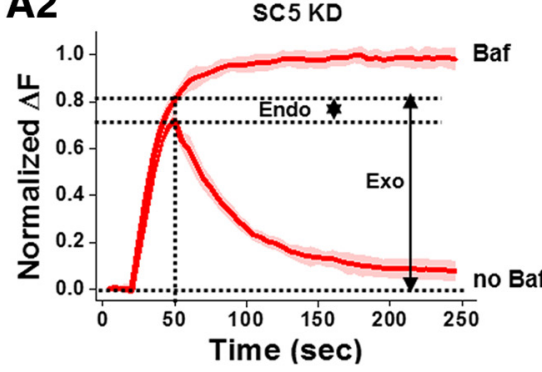

A3

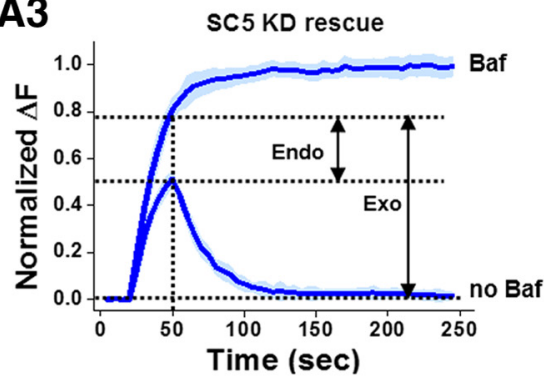

E

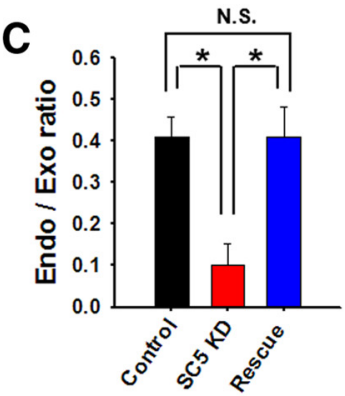

D

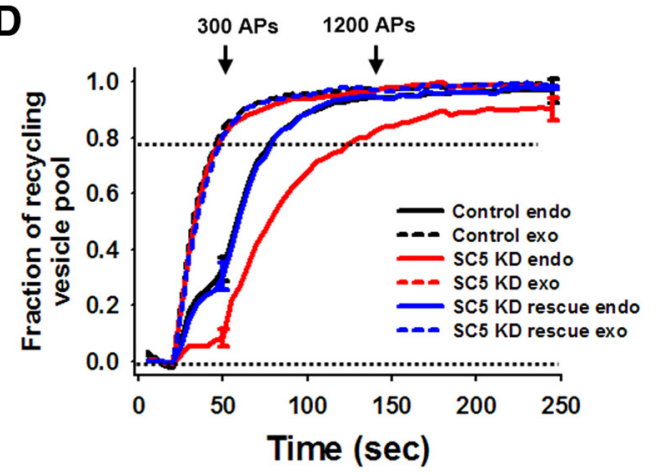

F1

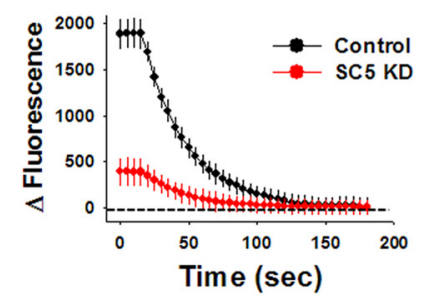

F2

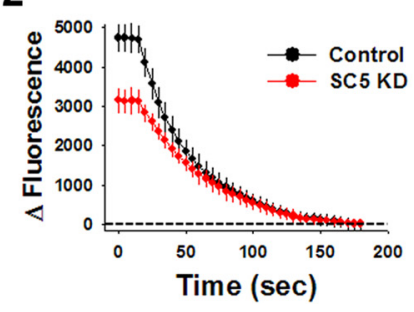

F3

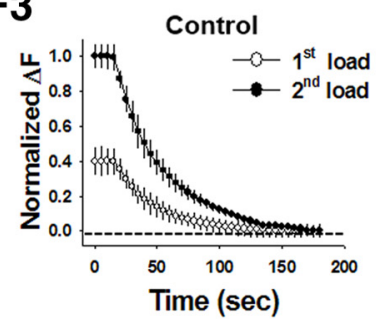

F4

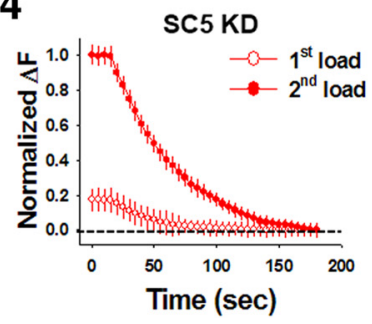

Figure 6. Independent verification of SCAMP5 KD-induced endocytic defects during stimulation using SypHy or FM 1-43. A1-A3, Average SypHy fluorescence intensity profiles from control (A1), KD (A2), and rescued (A3) synapses stimulated with or without Baf. Neurons were stimulated with $300 \mathrm{APs}$ at $10 \mathrm{~Hz}$ without Baf. After a 10 min resting period, the same neurons were stimulated again with $1200 \mathrm{APs}$ at $10 \mathrm{~Hz}$ in the presence of Baf. Fluorescence values were normalized to the maximal stable fluorescence signal in the Baf trace. $\boldsymbol{B}$, Decay of SypHy after stimulation fitted by a single exponential with time constant, $\tau=25.66 \pm 4.51 \mathrm{~s}$ for control $(n=8), 39.59 \pm 5.53 \mathrm{~s}$ for SCAMP5 KD $(n=10), 24.87 \pm 6.13 \mathrm{~s}$ for SCAMP5 KD rescue $(n=8)$. Data are presented as means \pm SE. ${ }^{*} p<0.01$ (ANOVA and Tukey's HSD post hoc test). C, Rate of exocytosis and rate of endocytosis during stimulation were obtained from the linear fits to the initial $30 \mathrm{~s}$ of traces (exocytic rate: $0.134 \pm 0.04$ for the control, $0.133 \pm 0.04$ for SCAMP5 KD, and $0.131 \pm 0.06$ for SCAMP5 rescue; endocytic rate: $0.055 \pm 0.03$ for the control, $0.0135 \pm 0.04$ for SCAMP5 KD, and $0.053 \pm 0.04$ for SCAMP5 rescue). The ratios of endocytosis/exocytosis rate: $0.410 \pm 0.04$ for the control, $0.101 \pm 0.05$ for SCAMP5 KD, and $0.408 \pm 0.07$ for SCAMP5 rescue. Data are presented as means \pm SE. ${ }^{*} p<0.01$ (ANOVA and Tukey's HSD post hoc test). $\boldsymbol{D}$, Average time course of endocytosis from control (black), KD (red), and rescue (blue) neurons during and after $300 \mathrm{APs}$ at $10 \mathrm{~Hz}$ without $B$ af and the average time course of exocytosis from control (black dotted), $K D$ (red dotted), and rescue (blue dotted) neurons during $1200 \mathrm{APs}$ at $10 \mathrm{~Hz}$ with Baf. The dashed line indicates the extent of exocytosis at the 30 s time point where the endocytosis to exocytosis ratios is calculated. Error bars are shown at two time points on the endocytosis curves. $E$, Experimental protocol used to compare endocytosis during stimulation and after stimulation using FM1-43. For endocytosis during stimulation, FM1- 43 was loaded at the onset of stimulation (300 APs) and immediately washed out with the cessation of stimulation. After a $10 \mathrm{~min}$ resting period, $1200 \mathrm{APs}$ at $10 \mathrm{~Hz}$ were given to unload and measure the amount of loaded FM1-43. The same neurons were stimulated again in the presence of FM1 - 43 and kept in the presence of dye for an additional $30 \mathrm{~s}$ after stimulation to label poststimulus endocytosed vesicles. After a $10 \mathrm{~min}$ resting period, $1200 \mathrm{APs}$ at $10 \mathrm{~Hz}$ were given to unload and measure the amount of loaded FM1-43. F1-F2, Pooled average traces of FM1- 43 signal of first load (F1) or second load (F2) from the similar size of boutons in control (black) and SCAMP5 KD (red) during 1200 AP stimulation (F1: $1894.33 \pm 169.97, n=8$ for control, $387.54 \pm 160.83, n=10$ for KD; $F 2: 4723.83 \pm 366.68$ for control, $3135 \pm 328.78$ for KD). F3-F4, Normalized average time course of FM1- 43 fluorescence intensity of first and second load during unloading with $1200 \mathrm{APs}$ at $10 \mathrm{~Hz}$. Fluorescence values were normalized to the maximal FM1- 43 fluorescence signal after second load (first load over second load: $0.401 \pm 0.082$ for control, $0.173 \pm 0.039$ for KD).

dent of presynaptic heterogeneity, we normalized the pool size to the total vesicle pool size. We found that the recycling/resting pool ratio in SCAMP KD cells was significantly increased by expanding the recycling fraction at the expense of the resting fraction (recycling fraction: resting fraction $=61.2 \pm 4.8 \%, 38.8 \pm$ $4.8 \%$ for the control; $80.1 \pm 5.7 \%, 19.9 \pm 5.7 \%$ for SCAMP5 KD; Fig. 2C1-C3). Overexpression of SCAMP5 did not affect the SV pool composition (Fig. 2D1-D3).

We next tested the effect of SCAMP5 KD on exo-endocytic trafficking of SVs. We found that SCAMP5 KD slowed endocy- tosis after stimulation to some extent $(300$ APs at $10 \mathrm{~Hz} ; \tau=$ $30.28 \pm 4.51$ for control, $58.96 \pm 4.82$ for KD; Fig. $3 A, B)$. When an HA-SCAMP5 that is resistant to shRNA was introduced into SCAMP5 KD neurons, the endocytic defect was fully rescued, indicating that the decrease in the rate of endocytosis was SCAMP5 specific $(\tau=31.75 \pm 3.19$ for rescue; Fig. $3 A, B)$.

The most striking effect of SCAMP5 KD was, however, on endocytic kinetics during stimulation. Because changes in fluorescence levels in the presence of Baf reflect pure exocytosis, whereas changes in the absence of Baf represent the balance be- 
A

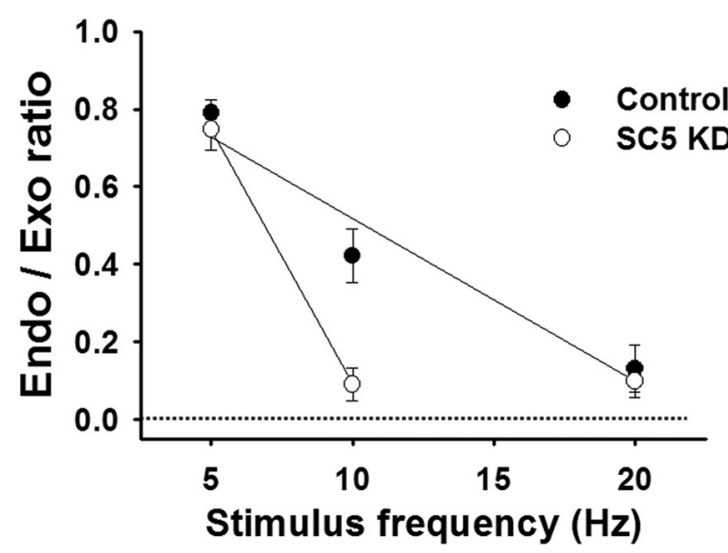

B

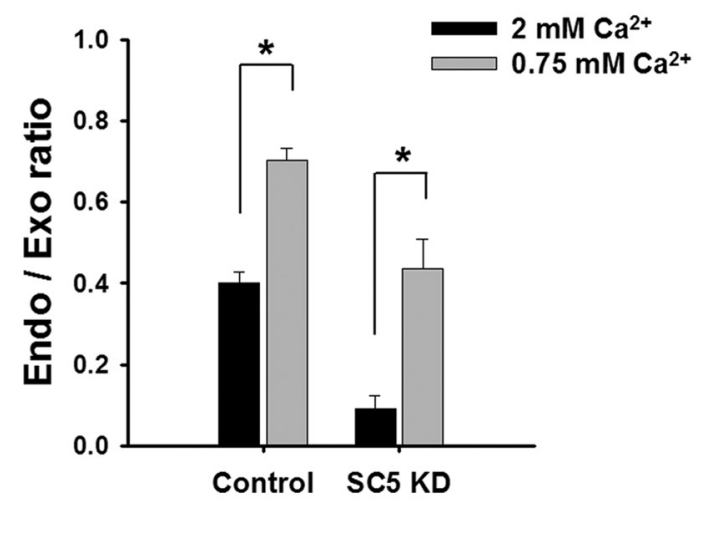

Figure 7. KD of SCAMP5 lowered the threshold of activity at which the SV endocytosis becomes unable to compensate for ongoing the exocytosis during the stimulus. $A$, Endo/Exo ratios after 300 APs at different frequencies. Dashed lines are linear fits (control: $\mathrm{KD}=0.790 \pm 0.03: 0.751 \pm 0.05 \mathrm{at} 5 \mathrm{~Hz}, 0.421 \pm 0.07: 0.091 \pm 0.04 \mathrm{at} 10 \mathrm{~Hz}, 0.131 \pm 0.06: 0.099 \pm 0.03$ at $20 \mathrm{~Hz}$ ). $n=8$ for control, $n=8$ for KD at each frequency. $\boldsymbol{B}$, Mean Endo/Exo ratios after $300 \mathrm{APs}$ at $10 \mathrm{~Hz}$ in $2 \mathrm{~mm}$ or $0.75 \mathrm{~mm}$ extracellular $\left[\mathrm{Ca}^{2+}\right]$. At $0.75 \mathrm{~mm}\left[\mathrm{Ca}^{2+}\right]$, Endo/Exo ratio: $0.701 \pm 0.03, n=5$ for control, $0.436 \pm 0.01, n=7$ for KD. Data are presented as means \pm SE. ${ }^{*} p<0.01$ (Student's $t$ test).

tween exocytosis and endocytosis during stimulation, the time course of endocytosis during stimulation could be estimated by simply subtracting the fluorescence values (Fernández-Alfonso and Ryan, 2004; Burrone et al., 2006). We found that in control synapses, $\sim 40 \%$ of the SVs that had undergone exocytosis had already been recovered by endocytosis during a stimulation of $300 \mathrm{APs}$ at $10 \mathrm{~Hz}$. In SCAMP5 KD neurons, however, $<10 \%$ of the exocytosed SVs had been recovered by endocytosis during that stimulus (Fig. 4A1-B3). The ratio of endocytosis/exocytosis during stimulation, calculated by obtaining the slopes after linear fitting of the time course of endocytosis and exocytosis to the 300 AP train, was significantly reduced in SCAMP5 KD neurons compared with controls (Fig. 4B4), pointing to severe endocytic defects in these cells. Again, the endocytic defects were fully rescued in cells containing HA-SCAMP5 resistant to shRNA (Fig. 4A1-B4). KD of SCAMP5 with an independent shRNA (shRNA\#1) gave rise to similar endocytic defects (Fig. 4C1-C3).

The endocytic defects observed during stimulation of SCAMP5 KD neurons became more evident when the average time courses of exocytosis and endocytosis in control and KD neurons were compared. There was no statistically significant difference between the average time course of exocytosis from control and KD neurons, indicating that the kinetics of SV exocytosis were not affected by SCAMP5 KD and that SCAMP5 functions in the endocytic pathway (Fig. 5A). A composite graph of the average time course of total endocytosis was obtained by combining the time course of endocytosis during stimulation [i.e., (+)Baf - (-)Baf] with the inverse image of the time course of endocytosis after stimulation (Fig. 4A1-A3). Compared with control and SCAMP5 KD-rescued neurons (Fig. 5A, black and blue line, respectively), SCAMP5 KD neurons (Fig. $5 A$, red line) showed considerable defects in endocytosis during stimulation, whereas poststimulus endocytosis was only mildly affected (Fig. $5 A$ ). With prolonged stimulation (900 APs at $10 \mathrm{~Hz}$ ), the endocytic defects in SCAMP5 KD synapses during stimulation were more pronounced than in the control synapses and virtually no exocytosed SVs were endocytosed (Fig. 5B). This difference was not due to defective acidification because, after a $30 \mathrm{~s}$ stimulus, fluorescence was quenched to the same extent as during the prestimulus period (average degree of quenching poststimulus:
$94.11 \pm 5.88 \%$ in control and $95.11 \pm 4.88 \%$ in $\mathrm{KD}$; Fig. $5 C 1-C 4)$.

To avoid a possible bias caused by an SV-protein-specific recycling mode (Hua et al., 2011; Raingo et al., 2012; Ramirez et al., 2012), SypHy, a fusion protein of synaptophysin with pHluorin (Granseth and Lagnado, 2008), was used. In agreement with the data obtained from vGpH-transfected neurons, SV endocytosis during stimulation was again severely defective in the SCAMP5 $\mathrm{KD}$ neurons and was fully rescued by the expression of HASCAMP5 resistant to shRNA (Fig. 6A-D), indicating that the SV retrieval defect caused by SCAMP5 KD is independent of the particular SV protein used.

To further eliminate any influence of an SV protein-specific endocytic mode on the analysis, FM1-43, a green fluorescent strylyl membrane dye that is widely used to study SV recycling kinetics (Betz and Bewick, 1992; Ryan et al., 1993; Ryan, 2001), was used. When FM1-43 was present during stimulation of SCAMP5 KD cells, the amount of endocytosed FM1-43 was considerably lower than that of control $(20.45 \pm 0.05 \%$ of the control), but after continued incubation with FM1-43 after stimulation, the difference was substantially reduced $(66.36 \pm$ $0.01 \%$ over control), again indicating that the severe endocytic defect was restricted to the period of stimulation (Fig. 6F1-F4).

Next, we plotted endocytosis/exocytosis ratios during stimulation of $300 \mathrm{APs}$ at different frequencies. We found that, at the lower stimulation frequency $(5 \mathrm{~Hz})$ SCAMP5 KD synapses did not show a significant endocytic defect (endocytosis/exocytosis ratio: $0.790 \pm 0.032$ for control, $0.747 \pm 0.054$ for SCAMP5 KD), indicating that SCAMP5 KD synapses performed endocytosis normally when the exocytosis burden was low (Fig. 7A). At $10 \mathrm{~Hz}$, however, SCAMP5 KD synapses displayed severe endocytic defects during stimulation (endocytosis/exocytosis ratio: $0.091 \pm$ 0.032; control: $0.421 \pm 0.07)$. At a higher stimulation frequency of $20 \mathrm{~Hz}$, the endocytosis/exocytosis ratio in control synapses was also decreased dramatically, suggesting that the cell's endocytic capacity was saturated with an increased stimulus frequency of 20 $\mathrm{Hz}$ (Fig. 7A). The inability of SCAMP5 KD neurons to match the rate of endocytosis to that of the ongoing exocytosis at $10 \mathrm{~Hz}$ was corroborated by the finding that, when extracellular $\left[\mathrm{Ca}^{2+}\right]$ was decreased to $0.75 \mathrm{~mm}$ to reduce the SV release probability (i.e., to 
A1

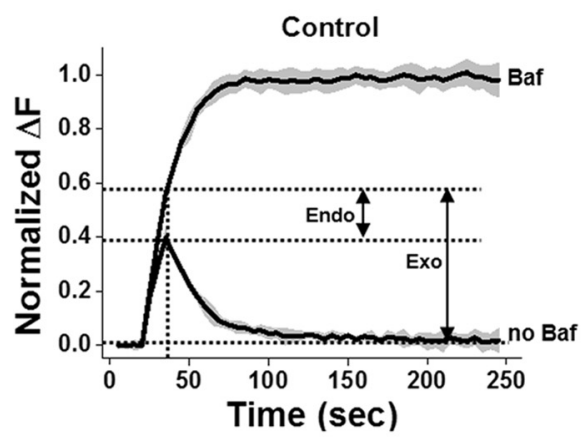

B1

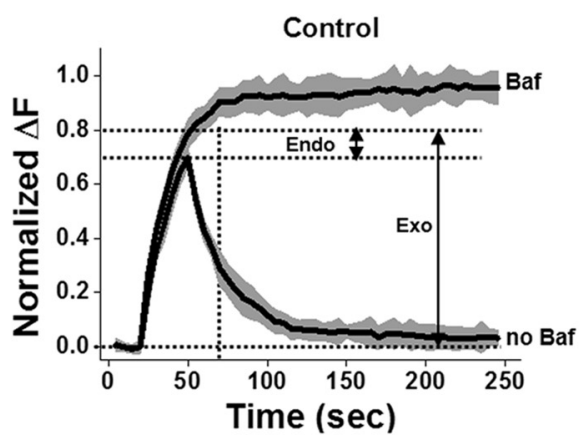

A2

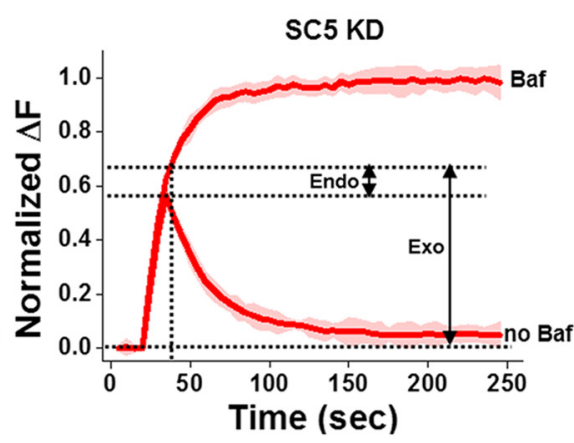

A3

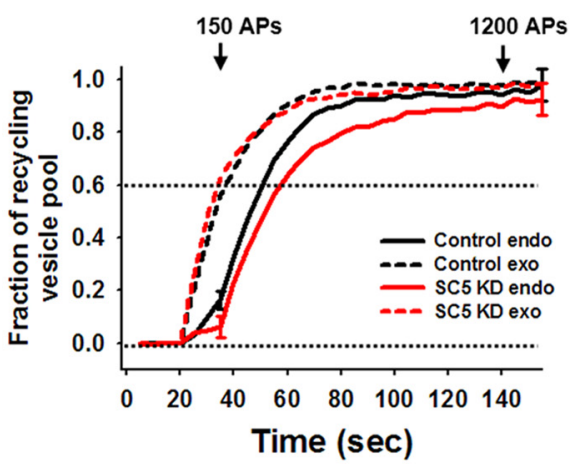

B3
B2

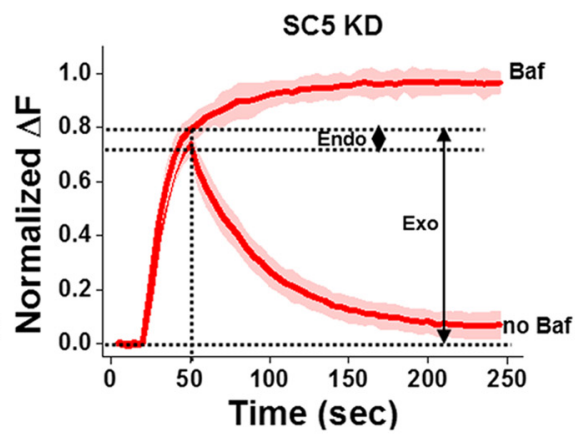

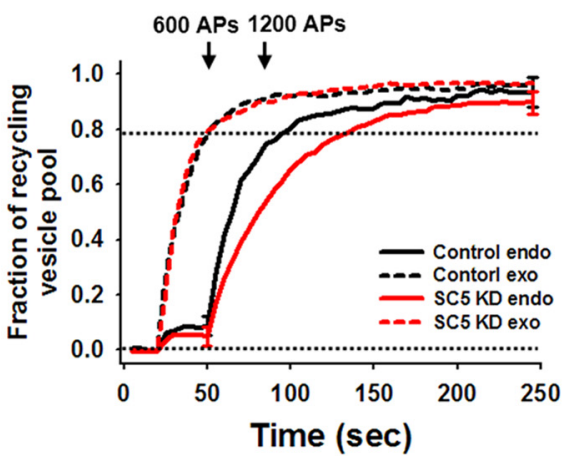

Figure 8. The frequency, not the duration, of stimulation was important for the observed defects in endocytosis in SCAMP5 KD. A1-A2, Average vGpH fluorescence intensity profiles from control (A1) and KD (A2) synapses stimulated with or without Baf. Neurons were stimulated at $10 \mathrm{~Hz}$ for $15 \mathrm{~s}$ (150 APs) without Baf. After a 10 min resting period, the same neurons were stimulated again with $1200 \mathrm{APs}$ at $10 \mathrm{~Hz}$ in the presence of $B$ af. Fluorescence values were normalized to the maximal stable fluorescence signal in the Baftrace. $n=9$ for control, $n=10$ for KD. $\mathbf{A 3}$, Average time course of endocytosis from control (black) and KD (red) neurons during and after $150 \mathrm{APs}$ at $10 \mathrm{~Hz}$ without Baf and the average time course of exocytosis from control (black dotted) and KD (red dotted) during $1200 \mathrm{APs}$ at $10 \mathrm{~Hz}$ with Baf. Error bars are shown at two time points on the endocytosis curves. $\boldsymbol{B 1}$ - B2, Average vGpH fluorescence intensity profiles from control (B1) and KD (B2) synapses stimulated with Baf or without $B$ af. Neurons were stimulated at $20 \mathrm{~Hz}$ for $30 \mathrm{~s}(600 \mathrm{APs})$ without $B$ af. After $10 \mathrm{~min}$ resting period, the sameneurons were stimulated again with $1200 \mathrm{APs}$ at $20 \mathrm{~Hz}$ in the presence of $B$ af. Fluorescence values were normalized to the maximal stable fluorescence signal in the Baftrace. $n=11$ for control, $n=11$ for KD. B3, Average time course of endocytosis from control (black) and KD (red) neurons during and after 600 APs at $20 \mathrm{~Hz}$ without Baf and the average time course of exocytosis from control (black dotted) and $\mathrm{KD}$ (red dotted) during $1200 \mathrm{APs}$ at $20 \mathrm{~Hz}$ with Baf.

reduce the exocytic load; Murthy et al., 1997; Trommershäuser et al., 2003), the endocytic defects of the SCAMP5 KD synapses were less severe at $10 \mathrm{~Hz}$ (endocytosis/exocytosis ratio: $0.436 \pm 0.007$ at $0.75 \mathrm{~mm}\left[\mathrm{Ca}^{2+}\right]$; Fig. $\left.7 B\right)$.

The endocytic defects of SCAMP5 KD synapses at $10 \mathrm{~Hz}$ were also observed with $15 \mathrm{~s}$ stimulation (150 APs; Fig. 8A1-A3). With a $30 \mathrm{~s} \mathrm{stimulation}$ at $20 \mathrm{~Hz}$ (600 APs), we again found that endocytic capacity was saturated in the control (Fig. 8B1-B3), as in the case with $15 \mathrm{~s}$ stimulation at $20 \mathrm{~Hz}$, suggesting that the frequency of stimulation (i.e., how fast exocytosis occurs so that how fast SVs accumulate on the plasma membrane over the endocytic capacity) is important. All of these results indicate that KD of SCAMP5 lowers the threshold of synaptic activity at which SV endocytosis becomes unable to compensate for ongoing exocytosis during stimulation.

\section{Discussion}

Most previous studies of the SCAMPs have focused on the regulation of exocytosis during DCV secretion or vesicle trafficking in the TGN. SCAMP1 plays a dual role in facilitating dilation and closure of fusion pores and has been implicated in exoendocytic coupling and in the regulation of DCV secretion in PC12 cells (Fernández-Chacón et al., 1999; FernándezChacón et al., 2000; Zhang and Castle, 2011). SCAMP2 is known to interact with Arf6, phospholipase D1, and phosphatidylinositol 4,5-bisphosphate via its E-peptide 2-3 cytoplasmic loop domain (CWYRPIYKAFR) and regulates fusion pore formation during DCV exocytosis (Guo et al., 2002; Liu et al., 2002; Liu et al., 2005; Liao et al., 2007). In addition, it interacts with the mammalian $\left(\mathrm{Na}^{+}, \mathrm{K}^{+}\right) / \mathrm{H}^{+}$exchanger NHE7 in the TGN and participates in the shuttling of NHE7 between recycling vesicles and the TGN (Lin et al., 2005). The NPF repeats of SCAMP1 are also known to bind to two EH domain proteins: intersectin 1, which is involved in endocytic budding at the plasma membrane, and $\gamma$-synergin, which may mediate the budding of vesicles in the TGN (Fernández-Chacón et al., 2000). Expression of SCAMP1 without the N-terminal NPF repeats potently inhibits transferrin uptake by endocytosis (Fernández-Chacón et al., 2000).

Compared with other SCAMPs, however, less is known about the role of SCAMP5. One recent study showed that its expression is markedly increased in the striatum of Huntington's disease patients and that its downregulation alleviates ER stress-induced protein aggregation in huntingtin mutants and the inhibition of endocytosis (Noh et al., 2009). Another study showed that human SCAMP5 interacts directly with synaptotagmin via its cytosolic C-terminal tail and is involved in calcium-regulated exocytosis of signal-peptide-containing cytokines (Han et al., 2009).

However, we found here that KD of SCAMP5 caused endocytic defects during strong stimulation of cultured hippocampal neurons, whereas exocytic kinetics were not affected. The difference in the effects of SCAMP5 could be due to mechanistic dif- 
ferences in exo-endocytosis between non-neuronal cells and neurons during intense stimulation. Unlike neurons, nonneuronal cells are never exposed to such strong activation in vivo. This explanation is consistent with the fact that $\mathrm{KD}$ synapses displayed little or no defects in endocytosis when stimulated at low frequency $(5 \mathrm{~Hz})$. It seems that, in SCAMP5 KD neurons, the endocytic capacity to cope with heavy exocytic loads is reduced, whereas the endocytosis activity of individual SVs during mild exocytic loads remains largely unaffected. In addition, although we did not find any defects in exocytosis in SCAMP5 KD synapses, we cannot completely rule out the possibility that SCAMP5 has an effect on exocytosis because the effect of SCAMP5 on single SV fusion kinetics was not tested. Instead, we tested the macroscopic kinetics of exocytosis upon sustained stimulation and this stimulation might mask subtle changes in unitary SV fusion kinetics.

Although total SV pool size varies from bouton to bouton even in a single neuron, when we compared the pooled average amplitude of signals from control and SCAMP5 KD boutons of similar size, SCAMP5 KD resulted in a reduction of total SV pool size as well as of that of the recycling pool. The ratio of recycling/ resting pool size was, however, significantly increased. Because endocytosis after stimulation was only moderately affected and exocytosis kinetics were not affected by SCAMP5 KD, we speculate that the change in this ratio could be due to a compensatory mechanism to allow neurons to maintain synaptic transmission during high levels of activity.

Our results further suggest that SCAMP5 is essential when neuronal activity is high and a heavy endocytic load is imposed on the cell. This is reminiscent of a study on the selective requirement for dynamin-1 during high levels of neuronal activity (Ferguson et al., 2007). The authors of that study found that dynamin-1 was dispensable for the endocytic recycling of SVs but became essential when an intense stimulus imposed a heavy load on endocytosis (Ferguson et al., 2007). In addition, a recent study showed that synaptophysin knock-out $\left(s y p^{-1-}\right)$ synapses exhibit defective SV endocytosis both during and after neuronal activity, whereas exocytosis and the size of the total recycling pool of SVs were unaffected (Kwon and Chapman, 2011). That study also found that $s y p^{-1-}$ neurons displayed pronounced synaptic depression and slower recovery of the recycling SV pool after depletion. Interestingly, synaptophysin and SCAMP5, together with synaptogyrin, are tetraspan vesicle membrane proteins (TVPs) because they have four transmembrane regions and cytoplasmically located termini (Hübner et al., 2002). Although previous studies found little or no phenotypic defects in neurons of knockout mice lacking synaptophysin, synaptogyrin-1, or SCAMP-1 (Eshkind and Leube, 1995; McMahon et al., 1996; FernándezChacón et al., 1999; Janz et al., 1999), our current data strongly suggest that the TVPs on SVs contribute to subtle neuronal functions such as the control of SV recycling during sustained neuronal activity.

SCAMP5 does not contain an N-terminal NPF motif and is not known to interact with dynamin-1 directly, but we have found that there are putative AP-2-binding sites (YXX $\phi)$ in its $\mathrm{N}$ terminus and 2-3 loop region. Therefore, SCAMP5 may interact with dynamin-1 and other endocytic proteins directly or indirectly to effectively accomplish endocytosis during intense stimulation. Further studies should focus on the molecular mechanisms through which SCAMP5 interacts with other endocytic proteins to control SV recycling.

In conclusion, SCAMP5 functions to control the SV recycling machinery when neuronal activity is high enough to impose a heavy load on endocytosis. It may recruit or promote the assembly of endocytic components to maintain an adequate number of endocytic machines during sustained neuronal activity. Our data support recent suggestions that changes in the expression of SCAMP5 in Huntington's disease and autism may be related to the synaptic dysfunction observed in these patients.

\section{References}

Balaji J, Ryan TA (2007) Single-vesicle imaging reveals that synaptic vesicle exocytosis and endocytosis are coupled by a single stochastic mode. Proc Natl Acad Sci U S A 104:20576-20581. CrossRef Medline

Betz WJ, Bewick GS (1992) Optical analysis of synaptic vesicle recycling at the frog neuromuscular junction. Science 255:200-203. CrossRef Medline

Burrone J, Li Z, Murthy VN (2006) Studying vesicle cycling in presynaptic terminals using the genetically encoded probe synaptopHluorin. Nat Protoc 1:2970-2978. Medline

Castermans D, Volders K, Crepel A, Backx L, De Vos R, Freson K, Meulemans S, Vermeesch JR, Schrander-Stumpel CT, De Rijk P, Del-Favero J, Van Geet C, Van De Ven WJ, Steyaert JG, Devriendt K, Creemers JW (2010) SCAMP5, NBEA and AMISYN: three candidate genes for autism involved in secretion of large dense-core vesicles. Hum Mol Genet 19:1368-1378. CrossRef Medline

Chang S, De Camilli P (2001) Glutamate regulates actin-based motility in axonal filopodia. Nat Neurosci 4:787-793. CrossRef Medline

Denker A, Rizzoli SO (2010) Synaptic vesicle pools: an update. Front Synaptic Neurosci 2:135. CrossRef Medline

Eshkind LG, Leube RE (1995) Mice lacking synaptophysin reproduce and form typical synaptic vesicles. Cell Tissue Res 282:423-433. CrossRef Medline

Ferguson SM, Brasnjo G, Hayashi M, Wölfel M, Collesi C, Giovedi S, Raimondi A, Gong LW, Ariel P, Paradise S, O'toole E, Flavell R, Cremona O, Miesenböck G, Ryan TA, De Camilli P (2007) A selective activitydependent requirement for dynamin 1 in synaptic vesicle endocytosis. Science 316:570-574. CrossRef Medline

Fernández-Alfonso T, Ryan TA (2004) The kinetics of synaptic vesicle pool depletion at CNS synaptic terminals. Neuron 41:943-953. CrossRef Medline

Fernández-Chacón R, Südhof TC (2000) Novel SCAMPs lacking NPF repeats: Ubiquitous and synaptic vesicle-specific forms implicate SCAMPs in multiple membrane-trafficking functions. J Neurosci 20:7941-7950. Medline

Fernández-Chacón R, Alvarez de Toledo G, Hammer RE, Südhof TC (1999) Analysis of SCAMP1 function in secretory vesicle exocytosis by means of gene targeting in mice. J Biol Chem 274:32551-32554. CrossRef Medline

Fernández-Chacón R, Achiriloaie M, Janz R, Albanesi JP, Südhof TC (2000) SCAMP1 function in endocytosis. J Biol Chem 275:12752-12756. CrossRef Medline

Granseth B, Lagnado L (2008) The role of endocytosis in regulating the strength of hippocampal synapses. J Physiol 586:5969-5982. CrossRef Medline

Guo ZH, Liu L, Cafiso D, Castle D (2002) Perturbation of a very late step of regulated exocytosis by a secretory carrier membrane protein (SCAMP2)derived peptide. J Biol Chem 277:35357-35363. CrossRef Medline

Han C, Chen T, Yang M, Li N, Liu H, Cao X (2009) Human SCAMP5, a novel secretory carrier membrane protein, facilitates calcium-triggered cytokine secretion by interaction with SNARE machinery. J Immunol 182:2986-2996. CrossRef Medline

Hua Z, Leal-Ortiz S, Foss SM, Waites CL, Garner CC, Voglmaier SM, Edwards RH (2011) v-SNARE composition distinguishes synaptic vesicle pools. Neuron 71:474-487. CrossRef Medline

Hübner K, Windoffer R, Hutter H, Leube RE (2002) Tetraspan vesicle membrane proteins: synthesis, subcellular localization, and functional properties. Int Rev Cytol 214:103-159. CrossRef Medline

Janz R, Südhof TC, Hammer RE, Unni V, Siegelbaum SA, Bolshakov VY (1999) Essential roles in synaptic plasticity for synaptogyrin I and synaptophysin I. Neuron 24:687-700. CrossRef Medline

Kwon SE, Chapman ER (2011) Synaptophysin regulates the kinetics of synaptic vesicle endocytosis in central neurons. Neuron 70:847-854. CrossRef Medline

Lee S, Lee K, Hwang S, Kim SH, Song WK, Park ZY, Chang S (2006) SPIN90/WISH interacts with PSD-95 and regulates dendritic spinogen- 
esis via an N-WASP-independent mechanism. EMBO J 25:4983-4995. CrossRef Medline

Liao H, Ellena J, Liu L, Szabo G, Cafiso D, Castle D (2007) Secretory carrier membrane protein SCAMP2 and phosphatidylinositol 4,5-bisphosphate interactions in the regulation of dense core vesicle exocytosis. Biochemistry 46:10909-10920. CrossRef Medline

Liao H, Zhang J, Shestopal S, Szabo G, Castle A, Castle D (2008) Nonredundant function of secretory carrier membrane protein isoforms in dense core vesicle exocytosis. Am J Physiol Cell Physiol 294:C797-C809. CrossRef Medline

Lin PJ, Williams WP, Luu Y, Molday RS, Orlowski J, Numata M (2005) Secretory carrier membrane proteins interact and regulate trafficking of the organellar $(\mathrm{Na}+, \mathrm{K}+) / \mathrm{H}+$ exchanger NHE7. J Cell Sci 118:18851897. CrossRef Medline

Liu L, Guo Z, Tieu Q, Castle A, Castle D (2002) Role of secretory carrier membrane protein SCAMP2 in granule exocytosis. Mol Biol Cell 13: 4266-4278. CrossRef Medline

Liu L, Liao H, Castle A, Zhang J, Casanova J, Szabo G, Castle D (2005) SCAMP2 interacts with Arf6 and phospholipase D1 and links their function to exocytotic fusion pore formation in PC12 cells. Mol Biol Cell 16:4463-4472. CrossRef Medline

McMahon HT, Bolshakov VY, Janz R, Hammer RE, Siegelbaum SA, Südhof TC (1996) Synaptophysin, a major synaptic vesicle protein, is not essential for neurotransmitter release. Proc Natl Acad Sci U S A 93:4760-4764. CrossRef Medline

Murthy VN, De Camilli P (2003) Cell biology of the presynaptic terminal. Annu Rev Neurosci 26:701-728. CrossRef Medline

Murthy VN, Sejnowski TJ, Stevens CF (1997) Heterogeneous release properties of visualized individual hippocampal synapses. Neuron 18:599612. CrossRef Medline

Noh JY, Lee H, Song S, Kim NS, Im W, Kim M, Seo H, Chung CW, Chang JW, Ferrante RJ, Yoo YJ, Ryu H, Jung YK (2009) SCAMP5 links endoplasmic reticulum stress to the accumulation of expanded polyglutamine protein aggregates via endocytosis inhibition. J Biol Chem 284:11318-11325. CrossRef Medline
Raingo J, Khvotchev M, Liu P, Darios F, Li YC, Ramirez DM, Adachi M, Lemieux P, Toth K, Davletov B, Kavalali ET (2012) VAMP4 directs synaptic vesicles to a pool that selectively maintains asynchronous neurotransmission. Nat Neurosci 15:738-745. CrossRef Medline

Ramirez DM, Khvotchev M, Trauterman B, Kavalali ET (2012) Vtila identifies a vesicle pool that preferentially recycles at rest and maintains spontaneous neurotransmission. Neuron 73:121-134. CrossRef Medline

Rizzoli SO, Betz WJ (2004) The structural organization of the readily releasable pool of synaptic vesicles. Science 303:2037-2039. CrossRef Medline

Rizzoli SO, Betz WJ (2005) Synaptic vesicle pools. Nat Rev Neurosci 6:57-69. CrossRef Medline

Ryan TA (2001) Presynaptic imaging techniques. Curr Opin Neurobiol 11: 544-549. CrossRef Medline

Ryan TA, Reuter H, Wendland B, Schweizer FE, Tsien RW, Smith SJ (1993) The kinetics of synaptic vesicle recycling measured at single presynaptic boutons. Neuron 11:713-724. CrossRef Medline

Sankaranarayanan S, Ryan TA (2000) Real-time measurements of vesicleSNARE recycling in synapses of the central nervous system. Nat Cell Biol 2:197-204. CrossRef Medline

Sankaranarayanan S, De Angelis D, Rothman JE, Ryan TA (2000) The use of pHluorins for optical measurements of presynaptic activity. Biophys J 79:2199-2208. CrossRef Medline

Trommershäuser J, Schneggenburger R, Zippelius A, Neher E (2003) Heterogeneous presynaptic release probabilities: functional relevance for short-term plasticity. Biophys J 84:1563-1579. CrossRef Medline

Voglmaier SM, Kam K, Yang H, Fortin DL, Hua Z, Nicoll RA, Edwards RH (2006) Distinct endocytic pathways control the rate and extent of synaptic vesicle protein recycling. Neuron 51:71-84. CrossRef Medline

Wu TT, Castle JD (1998) Tyrosine phosphorylation of selected secretory carrier membrane proteins, SCAMP1 and SCAMP3, and association with the EGF receptor. Mol Biol Cell 9:1661-1674. CrossRef Medline

Zhang J, Castle D (2011) Regulation of fusion pore closure and compound exocytosis in neuroendocrine PC12 cells by SCAMP1. Traffic 12:600614. CrossRef Medline 